\title{
Implementación del algoritmo MPC a un inversor de dos niveles para un motor trifásico de inducción
}

\author{
MPC algorithm implementation to a two-level inverter \\ for a three-phase induction motor
}

\author{
L.G. González, C. A. Espinoza, R. O. Guerrero
}

\begin{abstract}
The present work shows the design and implementation of a variable frequency drive for a three-phase induction motor using the model predictive control algorithm (MPC). The strategy is based on the estimation of the internal model machine to be controlled. The simulation analyzes the performance of the controller against variations in the estimation of the motor parameters at different operating points. The control system is implemented using the TMS320F28335 microcontroller and a three-phase two-level inverter using an IRAM 136 3063b. Finally, the performance of the experimental system is compared using computational tools.
\end{abstract}

Index Terms - Speed control, model predictive control, induction motor

Resumen-El presente trabajo muestra el diseño e implementación de un variador de frecuencia para un motor trifásico de tipo inducción utilizando el algoritmo de control predictivo por modelo (MPC). La estrategia se fundamenta en la estimación del modelo interno de la máquina a controlar. Mediante simulación se analiza el comportamiento del controlador ante variaciones en la estimación de los parámetros del motor y distintos puntos de operación. El sistema de control es implantado mediante el microcontrolador TMS320F28335 y un inversor trifásico de dos niveles con el uso del IRAM 136-3063b. Finalmente, el comportamiento del sistema experimental es comparado mediante herramientas computacionales.

Palabras Claves - Control de velocidad, control predictivo por modelo, motor de inducción

\section{INTRODUCCIÓN}

$\mathrm{E}^{\mathrm{n}}$ la actualidad, una gran variedad de procesos industriales presenta la necesidad de variar la velocidad de una máquina eléctrica. Para distintos fines como sistemas de bombeo de fluidos, bandas transportadoras y sistemas mecánicos en general. Una de las máquinas eléctricas más usadas en el sector industrial es el motor trifásico de inducción debido a su bajo costo y mantenimiento. Para el control de velocidad de máquinas de este tipo, una de las técnicas más usadas es basadas en la variación de la frecuencia [1], donde

L.G. Gonzalez, C. A. Espinoza and R. O. Guerrero are with the Departamento de Ingeniería Eléctrica, Electrónica y Telecomunicaciones, Universidad de Cuenca, Ecuador,(e-mail:\{luis.gonzalez@ucuenca.edu.ec, adrian.espinoza92@ucuenca.ec, ricardo.guerreroc@ucuenca.ec\}. muchos de los convertidores de potencia para tal fin utilizan técnicas de modulación que no optimizan el control y la entrega de energía, como por ejemplo la modulación por ancho de pulso sinusoidal (PWM) [1] en comparación a técnicas más recientes como las de modulaciones por espacios vectoriales (SVM) que aprovechan más el bus de continua y reducen las conmutaciones de los transistores [2].

El control de la velocidad de giro y par mecánico de un motor trifásico de inducción en el sector industrial cuenta con limitados métodos de control [3]. Los variadores de velocidad en su composición interna cuentan con convertidores de potencia encargados de transformar la energía en corriente continua a corriente alterna a frecuencia variable [3]. Para cumplir con este propósito, la implementación de un tipo de modulación es un aspecto importante. Actualmente, en la mayoría de implementaciones industriales los variadores de velocidad, inversores monofásicos o trifásicos utilizan modulación por ancho de pulso y modulación por espacios vectoriales pero hoy en días las técnicas basadas en control predictivo por modelo (MPC) tienen especial interés [4].

Otras técnicas con gran perspectiva son las que utilizan un control por modelo interno que utilizan estimaciones del proceso a controlar junto a horizontes de predicción que permiten mejorar el desempeño del sistema [6]. En este particular, una de estas técnicas destaca el MPC que permite hacer el seguimiento de las corrientes de referencia con la evaluación de las 8 combinaciones posibles en el inversor fuente de voltaje (VSI) de 2 niveles, esto con el fin de alcanzar la velocidad de operación deseada según las necesidades del proceso.

En el presente artículo se presenta el desarrollo de un variador de velocidad para un motor de baja potencia con el uso de la técnica de variación de frecuencia, ésta técnica se aplica con control digital desarrollado mediante un microcontrolador del tipo TMS320F28335 [7], de punto flotante, que ejecuta el control MPC desarrollado en [3]. En busca de mostrar la implementación de la técnica MPC con un motor de inducción y el desempeño del seguimiento de la velocidad del motor ante cambios en la referencia de frecuencia y error en las estimación en los parámetros de la carga a manejar, en el estudio se evidencia el comportamiento 
de la técnica tomando en cuenta considerables errores de los parámetros característicos de las maquinas eléctricas, con el uso de equipamiento que permite una rápida y versátil programación, que puede ser modificada para agregar características y ser utilizado un múltiples aplicaciones.

\section{Control Predictivo POR Modelo}

El MPC tiene su base en la utilización del modelo del sistema para predecir el comportamiento futuro de las variables controladas. El MPC se describe como una técnica de control general y no específico para un sistema determinado, por lo tanto, engloba una familia de controladores que desde los años 80 , han sido aplicadas y en los últimos años ha tenido especial interés en la electrónica de potencia [5]. Otras aplicaciones para el control de energía también han sido estudiadas en [8], [9].

El MPC para el control de velocidad de máquinas eléctricas mediante la variación de la frecuencia y amplitud de corriente, se describe como una estrategia basada en un número finito de estados posibles de conmutación que depende del nivel del convertidor [3], los cuales son usados para predecir el comportamiento de las variables de cada estado de conmutación. Esta técnica presenta la ventaja de no necesitar lazos de control sintonizables, a diferencia del control orientado a flujo, que utiliza generalmente lazos de control proporcionales integrales para el control de la corriente en la carga.

Para su implementación, el criterio consiste en una función de costo evaluada en todos los estados posibles del convertidor de potencia, para esta aplicación, dado el uso de un convertidor DC/AC como fuente de voltaje de dos niveles, los valores estimados de las corrientes de la carga se obtienen principalmente a partir de la medición de las corrientes actuales y del modelo de la carga, de manera que la predicción de los valores futuros se calcula para cada posible estado de conmutación y se selecciona el estado que minimiza el error mediante una función de costo [3]. El esquema que describe el MPC a utilizar es el usado en [3] que se muestra en la Fig. 1 y que es una modificación de la presentada en [2].

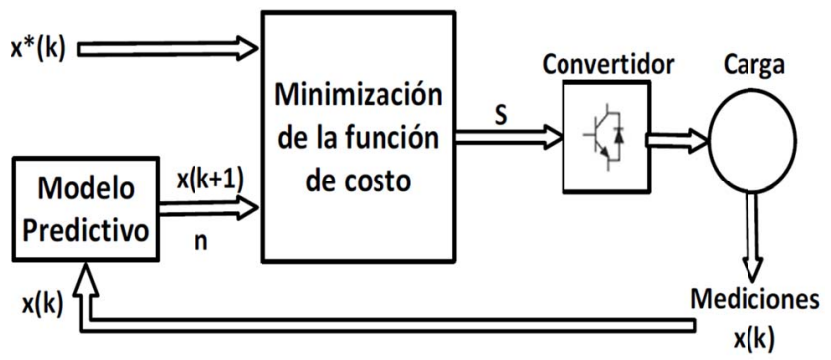

Fig. 1. Diagrama de bloques del lazo de control basado en MPC.

En la Fig. 1, $\mathbf{X}^{*}(k)$ es un vector de las corrientes de referencia en el instante $k$, donde a efectos del control de velocidad, estas impondrán la amplitud y frecuencia, $\mathbf{X}(k)$ es un vector de las mediciones de corriente en la carga, $\mathbf{X}(k+1)$ es el vector de predicción con un tamaño de $n=8$ posibles valores, y $\mathbf{S}$ es el vector de actuación óptima que se aplica al convertidor. La ecuación (1) representa el modelo típico de la carga a utilizar, donde $v_{N}, v_{a N}, v_{b N}, v_{c N}$ representan el voltaje de neutro y los voltajes de fase a neutro, respectivamente; $i_{a}, i_{b}, i_{c}$ representan las corrientes de línea; $e_{a}, e_{b}, e_{c}$ es la fuerza electromotriz inducida de la máquina; y, R y L representan la resistencia e inductancia equivalente por fase de la maquina [3]. A partir de la ecuación 1, se obtiene el modelo equivalente en el sistema estacionario ortogonal $(\alpha, \beta)$ con la transformada de Clarke, y a su vez al espacio discreto mediante la utilización de la aproximación de Euler hacia adelante en el cálculo de la derivada. Es importante destacar que el proceso de discretización se realiza a la frecuencia de muestreo, que es la misma de conmutación de los transistores $f_{s w}=40 \mathrm{kHz}$.

$$
\left[\begin{array}{l}
v_{a N} \\
v_{b N} \\
v_{c N}
\end{array}\right]=L \frac{d}{d t}\left[\begin{array}{c}
i_{a} \\
i_{b} \\
i_{c}
\end{array}\right]+R\left[\begin{array}{c}
i_{a} \\
i_{b} \\
i_{c}
\end{array}\right]+\left[\begin{array}{c}
e_{a} \\
e_{b} \\
e_{c}
\end{array}\right]+v_{N}
$$

Asumiendo que el sistema a controlar, es un sistema de tres hilos equilibrado donde $v_{N}=0$ y $e_{\alpha}(k) \approx e_{\alpha}(k+1)$ cuando $\left(f_{s w} \gg f\right)$, donde $f$ es la frecuencia fundamental de la corriente en los devanados de la maquina eléctrica y es variable en función a la velocidad de rotación deseada, se obtiene la corriente estimada de la componente $\alpha$ mediante (2) [3]:

$$
i_{\alpha}^{p}(k+1)=\left(1+\frac{R T_{s}}{L}\right) i_{\alpha}(k)+\frac{T_{s}}{L}\left[v_{\alpha}(k)-e_{\alpha}(k)\right]
$$

De la misma forma se obtiene la corriente estimada de la componente $\beta$, donde $i_{\alpha}^{p}$ representa la corriente estimada de línea de la componente $\alpha, i_{\alpha}$ es la corriente de línea medida en la componente $\alpha, T_{s}=1 / f_{s w}$ es el periodo de muestreo, $v_{\alpha}$ es el voltaje de línea en la componente $\alpha, e_{\alpha}$ es la fuerza electromotriz inducida de la máquina en la componente $\alpha$. La ecuación (2), utiliza las 8 combinaciones posibles que pueden estar presentes en el inversor trifásico de 2 niveles, estas combinaciones se identifican mediante el vector $\mathbf{S}$, compuesto por dos combinaciones nulas como (000) y (111) y 6 combinaciones activas (100) (110) (010) (011) (001) (101). Este vector permite estimar los posibles voltajes de salida $v_{\alpha}(k)$. Con el fin de minimizar el error entre las corrientes de referencia $\mathbf{X}^{*}(k)$ y las medidas es utilizada la función de costos según la ecuación (3) [3]:

$$
g=\left|i_{\alpha}^{*}(k+1)-i_{\alpha}^{p}(k+1)\right|+\left|i_{\beta}^{*}(k+1)-i_{\beta}^{p}(k+1)\right|
$$

Donde $i_{\alpha}^{*}$ e $i_{\beta}^{*}$ son las corrientes de referencia en la componente $\alpha$ y $\beta$, respectivamente, $i_{\alpha}^{p}$ e $i_{\beta}^{p}$ son las corrientes estimadas de línea de la componente $\alpha$ y $\beta$, respectivamente.

\section{CONSIDERACIONES TÉCNICAS DE DISEÑO}

La técnica de control en estudio es desarrollada en un convertidor AC/DC y DC/AC con potencia nominal de $3.3 \mathrm{~kW}$, a partir de una alimentación trifásica con tensión 
$V_{L L_{-} r m s}=220 \mathrm{~V}$ a $60 \mathrm{~Hz}$. Para cumplir con este objetivo se ha diseñado dos etapas definidas de la siguiente manera:

\section{A. Conversión $A C / D C$}

Esta etapa convierte la energía alterna del sistema trifásico a una corriente continua, en ella se utiliza un rectificador trifásico no controlado de onda completa, el dispositivo utilizado, corresponde a un rectificador no controlado modelo 36MT160. Con el fin de reducir el voltaje de rizo se utilizó un banco de condensadores con una capacidad nominal de $2 \mathrm{mF}$. Experimentalmente, se utilizaron dos condensadores en paralelo de $1000 \mu \mathrm{F}$, donde se obtiene un voltaje de rizado máximo del $5 \%$.

\section{B. Conversión DC/AC}

El convertidor de potencia destinado a generar la corriente alterna para la máquina eléctrica, utiliza el circuito dedicado del fabricante Infinieon $^{\circledR}$ de la serie i-Motion IRAM 136$3063 \mathrm{~b}$ [10], similar al utilizado en [11]. Este circuito contiene un convertidor con topología VSI de 2 niveles a base de transistores IGBTs con capacidad nominal de $3.3 \mathrm{~kW}$, corriente de rama máxima de $30 \mathrm{~A}$, voltaje máximo del bus DC de $600 \mathrm{~V}$ y es compatible con una señal TTL en entradas de control. En esta aplicación el circuito integrado cumple con las funciones de un inversor trifásico, además cuenta con circuitos de protección de sobre corriente y tensión en los transistores de potencia. Para la gestión de energía, el circuito cuenta con un total de 6 IGBTs en su interior que son activados por entradas lógicas con niveles compatibles a TTL.

El diagrama típico de conexión, es mostrado en la Fig. 2(a). De acuerdo al circuito típico de conexión se observa que los sistemas de excitación de los transistores superiores de rama utilizan un circuito de bombeo de carga (del inglés boot-strap) que permite energizar la unión puerta fuente. Para el dimensionamiento del circuito de excitación de puerta se ha fijado la frecuencia de conmutación en $f_{s w}=40 \mathrm{kHz}$, y condensadores de bombeo de carga de $4.7 \mu \mathrm{F}$. Con el uso del circuito antes mencionado el sistema de control de velocidad de la máquina eléctrica es presentado en la Fig. 2(b). El sistema de control es implementado en un controlador digital de la familia de microprocesadores TMS320F de Texas Instruments [7]. El control es utilizado con aislamiento galvánico mediante un optoacoplador de 6 canales unidireccionales para el manejo de las entradas de activación compatible con niveles TTL del IRAM 136-3063b.

En relación al sensado de corriente, se utilizan sensores de efecto hall del modelo FHS 40-P/SP600 [12] acoplados a circuitos de acondicionamiento de señal con filtros pasa bajo con frecuencias de corte en el orden de $530 \mathrm{~Hz}$, que permiten el aislamiento entre el sistema de control y potencia, y así reducir los problemas asociados a la interferencia electromagnética producida por la conmutación de los transistores en convertidor DC/AC.

Para lograr la variación de velocidad en la máquina eléctrica, el sistema utiliza corrientes de referencia con amplitud y frecuencia definidas sin tener realimentación de velocidad de la máquina, aspecto que permite variaciones en la velocidad debido al deslizamiento a medida que la máquina se exponga a cargas mecánicas en el rotor.

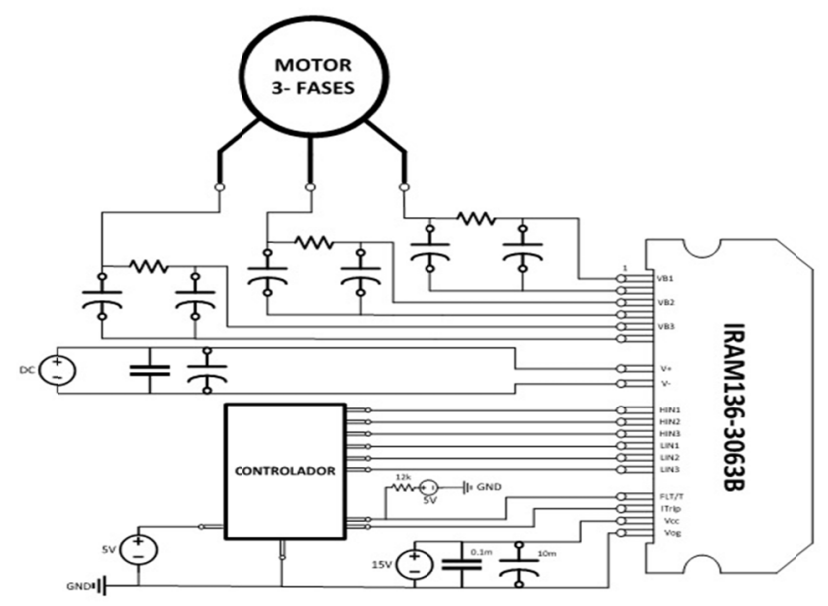

(a)

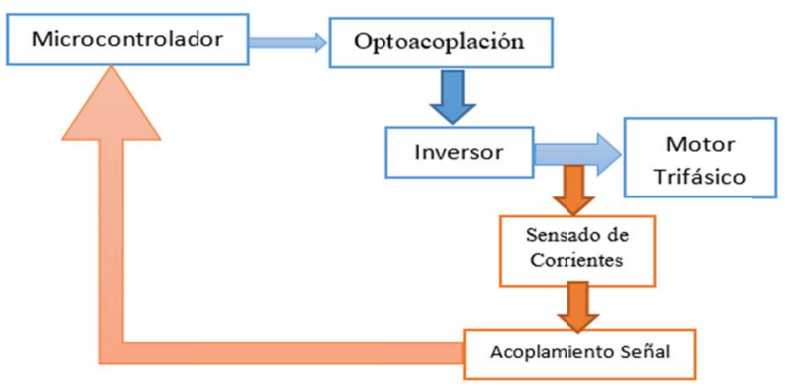

(b)

Fig. 2. (a) Conexión IRAM 136-3063b, (b) diagrama de bloques del variador de velocidad.

\section{ANÁLISIS DE COMPORTAMIENTO CON HERRAMIENTAS COMPUTACIONALES}

MPC al ser un algoritmo de alta eficiencia debe tener la capacidad de dar una respuesta efectiva sobre las variaciones que se puedan presentar en el sistema. En el presente apartado, se muestra un análisis de robustez del algoritmo MPC sobre la aplicación del variador de velocidad. La Fig. 3, muestra el diagrama emulado en PSIM $^{\circledR}$.

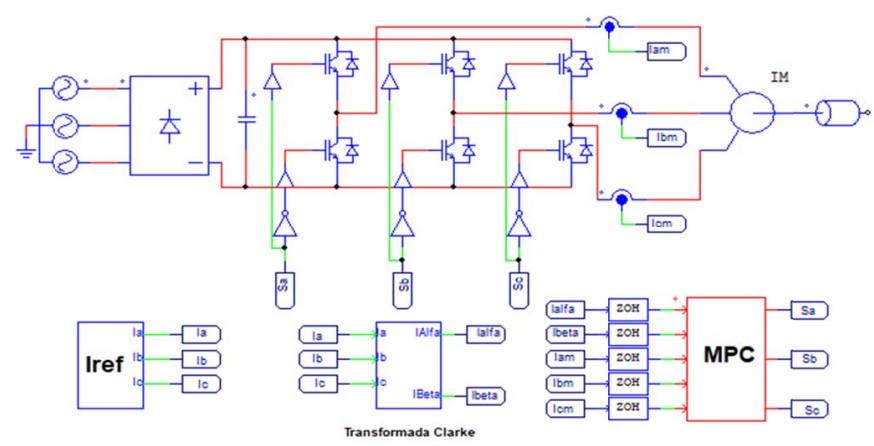

Fig. 3. Digrama control de velocidad bajo estudio emulado en $\operatorname{PSIM}^{\circledR}$

Para el control de velocidad del motor eléctrico se utilizada el convertidor de voltaje AC/DC, cuya señal no es ideal, y 
existe un rizado como se observa en la Fig. 4(a), es importante destacar que la frecuencia de rizado es de $360 \mathrm{~Hz}$ producto de la frecuencia de la fuente de alimentación y su componente espectral también se encuentra en el rizado de voltaje de salida en la carga. Una vez obtenido un voltaje en DC, la etapa de conversión DC/AC con el uso del algoritmo MPC permite obtener corrientes en el devanado de la máquina eléctrica, como la observada en la Fig. 4(b). Por medio del programa de simulación $\operatorname{PSIM}^{\circledR}$, con $L=6.41 \mathrm{mH}$ y $R=1.25 \Omega$, con un tiempo de muestro para el cálculo de la acción de control de $20 \mu \mathrm{s}$, en donde se observa que la distorsión armónica, que es del $6.63 \%$, esta distorsión no es significativa, si se considera que es una aplicación aislada y no está sujeto a una normativa en cuanto a calidad de energía, además con un seguimiento de la corriente de referencia con error instantáneo menor al 9\% y en promedio menor al $0.1 \%$.

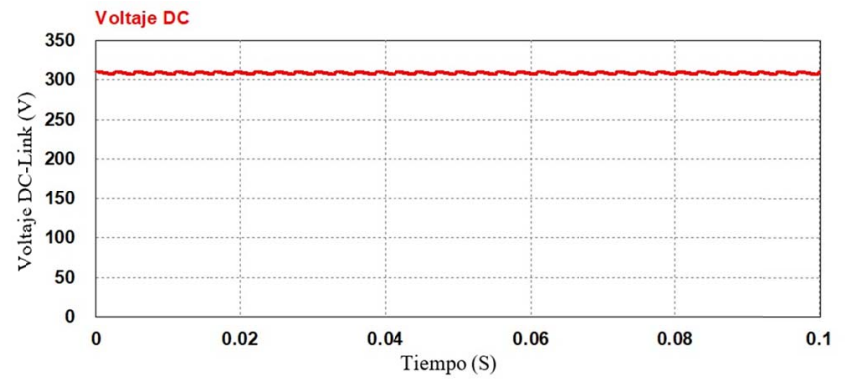

(a)

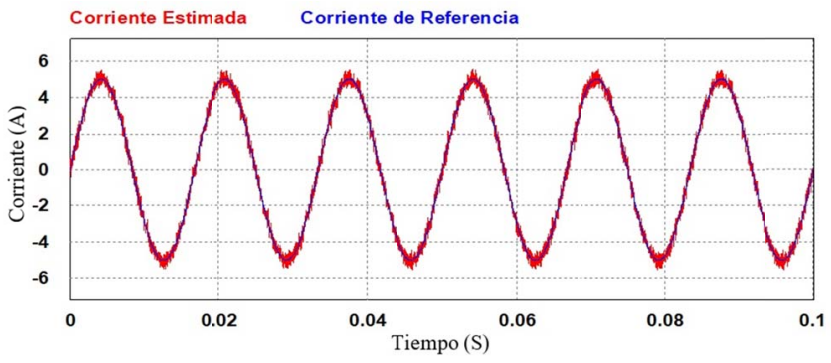

(b)

Fig. 4. (a) Comportamiento voltaje bus de continua, (b) Comportamiento del seguimiento de corriente de referencia

\section{A. Comportamiento ante variaciones en la estimación de los parámetros del motor}

Otro aspecto importante para comprobar la robustez del MPC sobre un sistema funcional, es analizar el comportamiento del mismo ante la estimación errada de los componentes internos del motor trifásico de inducción. Se han estudiado distintos escenarios, uno de ellos destaca mantener la resistencia de estator constante y asumir un error en la estimación de la inductancia de los devanados de la maquina a controlar en un $\pm 20 \%$. Las Fig. 5(a) y Fig. 5(b), muestran el comportamiento del seguimiento en la corriente de referencia, con amplitud de $5 \mathrm{~A}$ pico a $60 \mathrm{~Hz}$ con una variación del $+20 \%$ de la inductancia nominal para la Fig. 5(a) y $-20 \%$ en la Fig. 5 (b), en las figuras se observan el seguimiento de las corrientes con errores instantáneos menores al 10\%, en promedio menores al $0.1 \%$, adicionalmente estas corrientes tienen una distorsión armónica total de $6.5 \%$ y $7.22 \%$ respectivamente, distorsiones similares a las encontradas en [6].

Otro comportamiento estudiado, corresponde a la variación de $\pm 20 \%$ en la resistencia del estator. Las Fig. 6(a) y Fig. 6(b), muestran el comportamiento del seguimiento de la corriente de referencia con amplitud de $5 \mathrm{~A}$ pico a $60 \mathrm{~Hz}$ con una variación del $\pm 20 \%$ en la resistencia nominal $\mathrm{R}=1.25 \Omega$. Del comportamiento se obtienen errores instantáneos menores al $10 \%$, en promedio menores al $0.08 \%$, adicionalmente estas corrientes tienen una distorsión armónica total de $6.39 \%$ y $6.80 \%$ respectivamente.

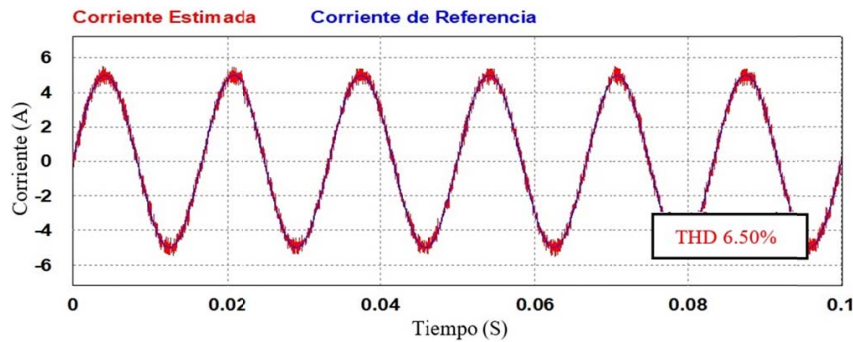

(a)

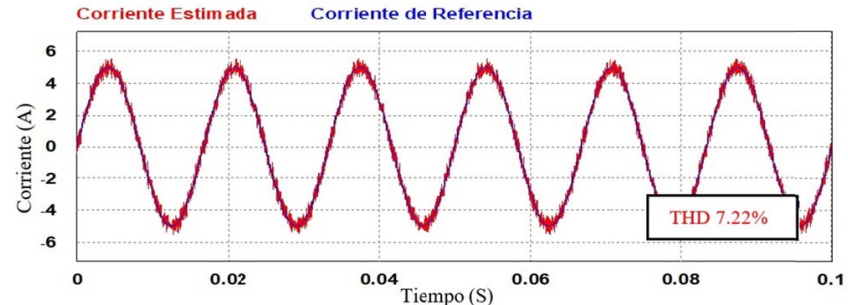

(b)

Fig. 5. Comportamiento de corriente ante variación inductancia de estator $\pm 20 \%$, (a) $+20 \% L=7.69 \mathrm{mH}$, (b) $-20 \%, L=5.34 \mathrm{mH}$.

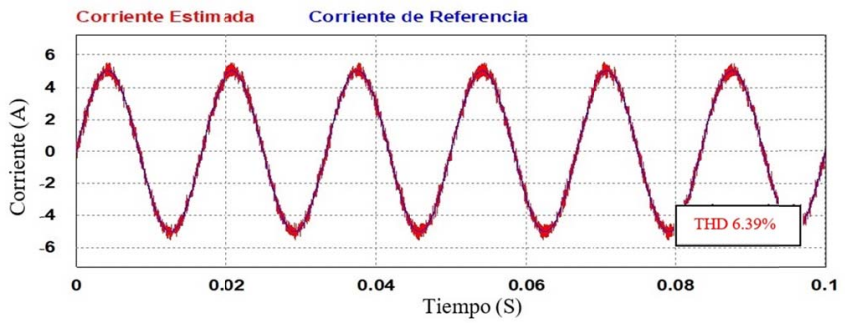

(a)

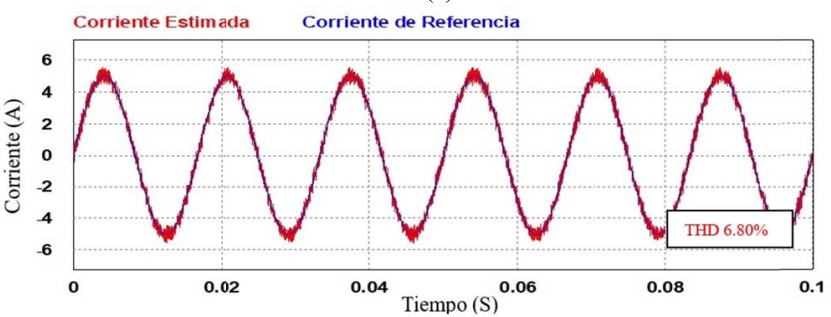

(b)

Fig. 6. Variación resistencia de estator $\pm 20 \%$, (a) $+20 \% R=1.5 \Omega$, (b) $-20 \%$, $R=1.04 \Omega$

B. Configuración de prototipo y análisis de desempeño experimental

El prototipo de control de velocidad del motor de inducción 
con la aplicación la técnica de control MPC se presenta en las Fig. 7(a) y Fig. 7(b), donde se muestra el circuito del convertidor de potencia $\mathrm{AD} / \mathrm{DC}$ y $\mathrm{DC} / \mathrm{AC}$ compuesto por el rectificador no controlado e IRAM 136-3063b respectivamente. En la Fig. 7(b), se observa el equipo de prueba donde se integra el sistema de control, convertidor de potencia y máquina eléctrica. Para la verificación del funcionamiento del sistema, se realizaron pruebas a lazo abierto con el fin de comprobar el funcionamiento de los siguientes componentes del sistema.

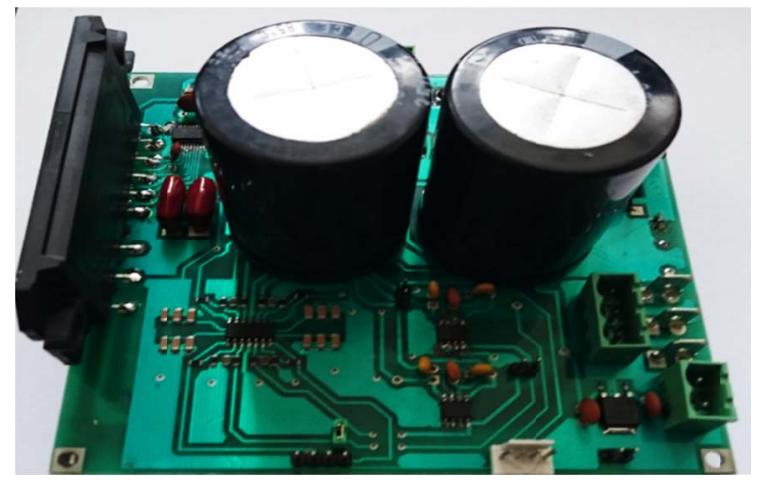

(a)

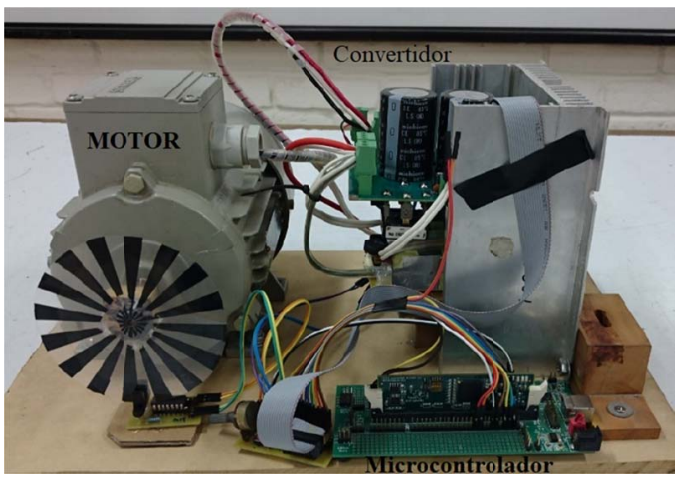

(b)

Fig. 7. Equipo de pruebas (a) vista superior convertidor $\mathrm{AC} / \mathrm{DC}$ y $\mathrm{DC} / \mathrm{AC}$, (b) banco de pruebas, motor + convertidor+microcontrolador

\section{Calibración y puesta en funcionamiento de instrumentación y acondicionamiento de señal}

Con la finalidad de evaluar el comportamiento del lazo de control por modelo predictivo, es necesario la calibración y comprobación del funcionamiento del conjunto de sensores dispuestos para tal fin, en el prototipo del convertidor se han utilizado únicamente sensores de efecto hall modelo FHS 40-P/SP600 para el sensado de dos corrientes de línea en la máquina a controlar. Dado que el sistema está compuesto por un sistema trifásico balanceado es posible estimar la corriente que circula por la línea no medida, aspecto que permite reducir el número de componentes en el sistema de sensado, y por ende los costos de su implementación. Con este procedimiento se alimentó el motor de inducción mediante la red eléctrica trifásica con una tensión línea a línea de $220 \mathrm{~V}$ y frecuencia nominal de $60 \mathrm{~Hz}$, bajo esta condición de operación, se observó el comportamiento de las corrientes de línea en la máquina eléctrica a partir del sensado y acondicionamiento de señales de las corrientes de la fase a y b. Finalmente se obtuvo una resolución del sensor de corriente de $26 \mathrm{mV} / \mathrm{A}$, y un valor de offset de $2.5 \mathrm{~V}$, aspecto importante para efectos de la ganancia de sensado en el sistema de control.

\section{Comportamiento del Sistema de CONTROL MPC}

Las pruebas de control a lazo cerrado hacen referencia a la aplicación experimental del control MPC a un motor de inducción trifásico con potencia nominal de $1 \mathrm{hp}$, modelo 3-MOTOR 1LA7 080-4YA60 marca SIEMENS ${ }^{\circledR}$, una vez comprobado el comportamiento de las etapas de sensado y aislamiento entre las etapas de control y potencia, se verificó el funcionamiento de la placa electrónica a través de la aplicación de un modulador PWM a lazo abierto antes de su uso en el lazo de control con el MPC, esto con la intención de comprobar el funcionamiento de los circuitos de conmutación y sensado. El algoritmo del MPC es puesto en funcionamiento con los parámetros calculados del estator del motor $(L=3.3951 \mathrm{mH}$ y $R=2.65065 \Omega)$. Estos parámetros fueron calculados mediante el procedimiento de mediciones a rotor bloqueado y a rotor libre [13]. Con la finalidad de comprobar el funcionamiento del sistema de control en el microcontrolador, se impone una corriente de referencia con amplitud de $I_{\text {ref }}=4 \mathrm{~A}$ a una frecuencia de $f=61.4 \mathrm{~Hz}$. Con ésta consigna el funcionamiento del algoritmo MPC predice las condiciones de conmutación del convertidor de potencia y permite obtener una velocidad de rotación de 3675RPM ante este escenario. Desde el aspecto del gasto computacional, el algoritmo del MPC programado presenta una duración aproximada de $16 \mu \mathrm{s}$, tiempo medido en una salida discreta que cambia de estado al inicio y final de la rutina de control; es importante destacar que el tiempo máximo de ejecución de la rutina es de los $25 \mu \mathrm{s}$, definido a partir de la frecuencia de conmutación utilizada. Es importante optimizar el programa del microcontrolador en este tipo de aplicaciones dado que generalmente la mayor cantidad de tiempo está asociada a las operaciones trigonométricas de la transformada de Clarke aplicada. La Fig. 8, presenta el comportamiento de la fase a del motor de inducción, variable medida con el circuito de sensado que representa una corriente pico de $I=4.03 \mathrm{~A}$.

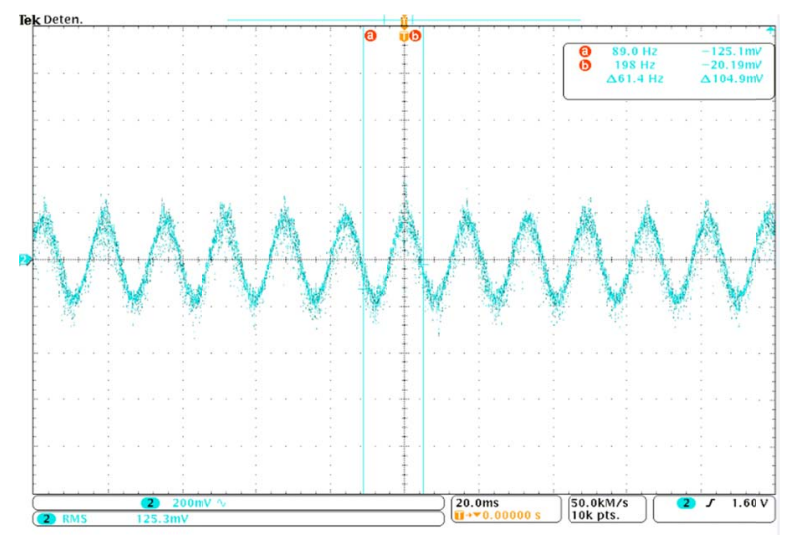

Fig. 8. Corriente de fase A aplicando MPC con $f=61.4 \mathrm{~Hz}$. 
La corriente obtenida es el resultado del sistema de control luego de la predicción establecida por el modelo de control predictivo. La distorsión armónica de la corriente medida con la aplicación osciloscopio es del $7.53 \%$, cerca de un $13 \%$ más del obtenido mediante simulación. El sistema de control realizado permite el control de velocidad de giro del motor mediante la variación de la frecuencia de la corriente entregada al motor. El algoritmo planteado permite la variación de frecuencia desde $37 \mathrm{~Hz}$ hasta $61.5 \mathrm{~Hz}$, esta referencia se ha establecido mediante una entrada análoga al microcontrolador TMS320F28335. En el caso aplicado en la Fig. 8, se muestra una frecuencia de operación de $f=61.4 \mathrm{~Hz}$ que representa la máxima velocidad de operación. En caso contrario, para frecuencias de referencia de $f=37 \mathrm{~Hz}$. La Fig. 9(a), muestra el comportamiento en régimen permanente a la menor frecuencia de operación donde se obtienen una velocidad de rotación de 2210RPM. La Fig. 9(b), muestra la predicción de corriente a una frecuencia intermedia $f=45.3 \mathrm{~Hz}$, donde el motor presenta una velocidad de rotación de 2710RPM.

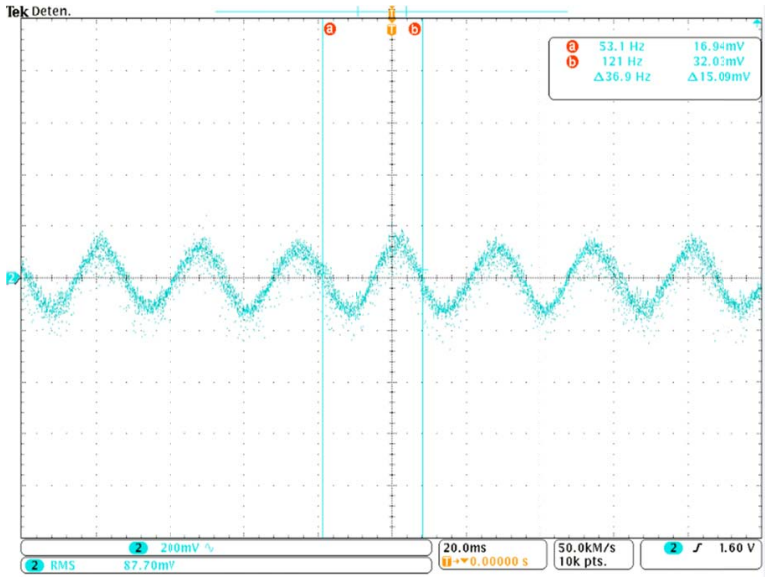

(a)

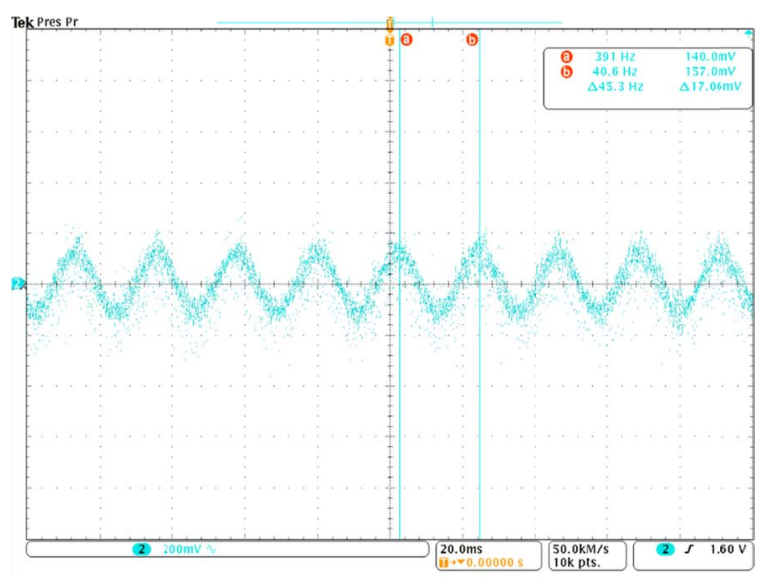

(b)

Fig. 9. Corriente de fase A aplicando MPC, (a) con $f=36.9 \mathrm{~Hz}$, (b) con $f=45.3 \mathrm{~Hz}$.

Por la naturaleza del comportamiento de un motor de inducción, la variación de velocidad no es directamente relacionada con la frecuencia de la corriente aplicada en el motor, este depende el factor de deslizamiento que este a su vez está relacionado con la carga mecánica al cual está sometida la máquina. En esta aplicación no se realizó ninguna compensación para tomar en cuenta este fenómeno, por lo que la frecuencia de referencia es manipulada en cualquier momento a través de una entrada análoga al microcontrolador mediante una resistencia variable.

La Fig. 10(a), presenta el comportamiento del control de velocidad, en RPM, al arranque del motor. Este comportamiento es similar a la respuesta de un sistema de primer orden ante una entrada escalón. De esta figura se observa, que el punto de régimen permanente se sitúa en 3600RPM (velocidad nominal del motor) y un tiempo de establecimiento cercano a $\mathrm{T}_{\mathrm{SS}}=1.6 \mathrm{~s}$. La Fig. 10(b), muestra el comportamiento dinámico del sistema de control de velocidad, datos reales mediante la adquisición de datos en tiempo real del microcontrolador TMS320F28335 con una duración del tiempo de muestreo de $25 \mu \mathrm{s}$, el comportamiento obtenido permite alcanzar a la consigna del lazo de control en tiempos similares en torno a $1.8 \mathrm{~s}$ sin presentar errores en estado estacionario.

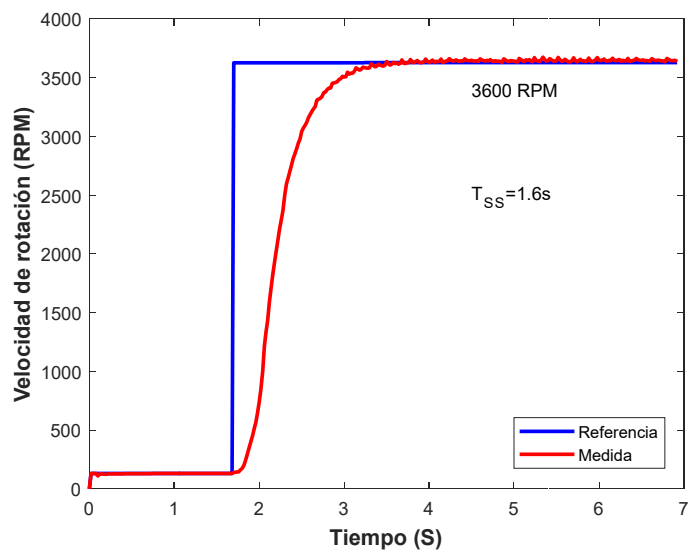

(a)

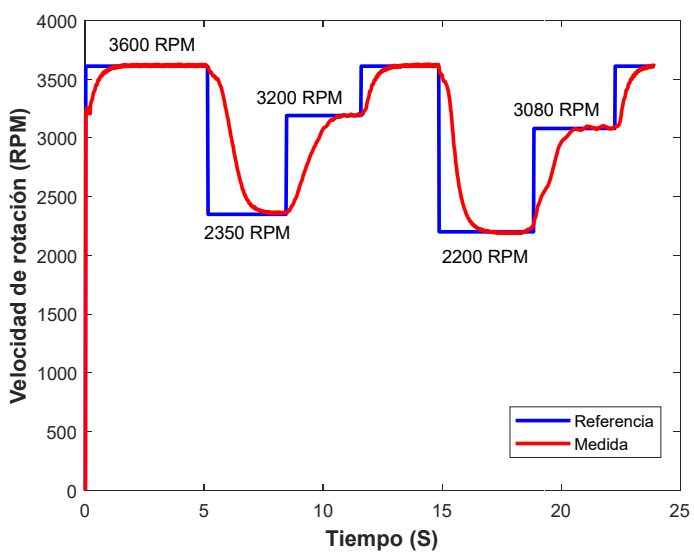

(b)

Fig. 10. (a) Comportamiento experimental ante arranque a velocidad nominal, (b) Comportamiento ante cambio de consigna.

\section{CONCLUSIONES}

El presente artículo muestra la implementación de un 
variador de frecuencia para motores asíncronos de inducción, utilizando la técnica de control por modelo predictivo. El algoritmo es implementado en un microcontrolador de punto flotante de altas prestaciones operando de forma aislada galvánicamente con la etapa de conversión de energía, en el mismo se estudian las capacidades del algoritmo de operar bajo errores en la estimación de los parámetros internos de la máquina, probando la robustez de la técnica hasta una estimación de los parámetros de la carga en un $20 \%$. Con base a los resultados obtenidos en simulaciones y mediante experimentación, el MPC predice las corrientes de la carga en base a la estimación del modelo interno de la máquina eléctrica, logrando un seguimiento de la velocidad de consigna de forma satisfactoria sin errores de estado estacionario. La implementación sirve cono referencia para realizar pruebas de técnicas de control en máquinas eléctricas, así como aplicaciones en convertidores de potencia aplicados a las energías renovables, específicamente a energía eólica gracias a la condición del bidireccionalidad de la topología utilizada.

\section{REFERENCIAS}

[1] M. A. K. A. Biabani and S. M. Ali, "Control of induction motor drive using space vector PWM," in Proc. ICEEOT 2016, Chennai, India, Mar. 2016, pp. 3344-3351.

[2] P. S. B. Sakti and S. Riyadi, "Hardware Implementation of Simplified VVVF Inverter for Induction Motor Based on SVM," in iSemantic 2019, Semarang, Indonesia, 2019, pp. 487-491.

[3] F. Wang, X. Mei, J. Rodriguez, and R. Kennel, "Model predictive control for electrical drive systems-an overview," Trans. Electr. Mach. Syst., vol. 1, no. 3, pp. 219-230, Sep. 2017.

[4] K. Wróbel, P. Serkies, and K. Szabat, "Model Predictive Base Direct Speed Control of Induction Motor Drive-Continuous and Finite Set Approaches," Energies, vol. 13, no. 5, p. 1193, Mar. 2020.

[5] S. Borreggine, V. G. Monopoli, G. Rizzello, D. Naso, F. Cupertino, and R. Consoletti, "A review on model predictive control and its application in power electronics," in Proc. AEIT AUTOMOTIVE 2019, Turin, Italy, 2019, pp. 1-6.

[6] Y. Zhang, B. Xia, H. Yang and J. Rodriguez, "Overview of model predictive control for induction motor drives", in Chinese Journal of Electrical Engineering, vol. 2, no. 1, pp. 62-76, Jun. 2016.

[7] Texas Instrument, "Product Folder Order Now TMS320F2833x, TMS320F2823x Digital Signal Controllers (DSCs) 1 Device Overview," 2007. [Online]. Available: www.ti.com. Accessed on: May 26, 2020.

[8] Y. Zhang, Y. Peng and C. Qu, "Model Predictive Control and Direct Power Control for PWM Rectifiers With Active Power Ripple Minimization," in IEEE Transactions on Industry Applications, vol. 52, no. 6, pp. 4909-4918, Nov.-Dec. 2016.

[9] M. Rivera et al., "A modulated model predictive control scheme for a two-level voltage source inverter," in Proc. ICIT 2015, Seville, Spain, 2015, pp. 2224-2229.

[10] "International Rectifier - IRAM136-3063B | 30A, 600V Integrated Power Module with internal shunt resistor." [Online]. Available: http://www.irf.com/part/30A-600V-INTEGRATED-POWERMODULE-WITH-INTERNAL-SHUNT-RESISTOR//A IRAM1363063B. Accessed on: May 26, 2020.

[11] S. Benavides-Córdoba et al. "Implementation of a Distribution Static Compensator D-STATCOM: Hardware and Firmware Description," Sci. Tech., vol. 24, no. 4, pp. 555-565, Dic. 2019.

[12] LEM, “Current transducer FHS 40-P/SP600," 2010. [Online]. Available moz-extension://28db55c8-a632-4aee-afcd-69a05257d468/enhancedreader.html?openApp\&pdf=https\%3A\%2F\%2Fwww.lem.com\%2Fsites $\% 2$ Fdefault $\% 2$ Ffiles $\% 2$ Fproducts_datasheets $\% 2 \mathrm{Ffhs} \% 252040$ p\%2520sp600.pdf. Accessed on: Jun 06, 2020.
[13] Y. Abdel-Rehim, "Parameter identification of induction motor," M.S. thesis, Dept. Computer Science and Electrical Engineering, West Virginia Univ., USA, 2015. 